\title{
THE PARTITION OF MULTI-RESOLUTION LOD BASED ON QTM
}

\author{
HOU Miao-le ${ }^{1}$, Xing Hua-qiao ${ }^{1}$, ZHAO Xue-sheng ${ }^{2}$, CHEN Jun ${ }^{3}$ \\ 1. Department of Computer Science, Beijing Institute of Civil Engineering and Architecture, Beijing 100044 China; \\ 2. China University of Mining and Technology in Beijing, Beijing 100083 China; \\ 3. National Geomatics Center of China, Beijing 100044 China
}

KEY WORD: Spherical Surface QTM, Multi- Resolution LOD, Viewpoint

\begin{abstract}
:
The partition hierarch of Quaternary Triangular Mesh (QTM) determine the accuracy of spatial analysis and application based on QTM. In order to resolve the problem that the partition hierarch of QTM is limited by the level of the computer hardware, the new method that Multi- Resolution LOD (Level of Details) based on QTM will be discussed in this paper. This method can make the resolution of the cells varying with the viewpoint position by partitioning the cells of QTM, selecting the particular area according to the viewpoint; dealing with the cracks caused by different subdivisions, it satisfies the request of unlimited partition in part.
\end{abstract}

About the first author: Hou Miaole, Female, 1974, Associate Professor of BUCEA who Get the PHD degree in 2005.7 at CUMTB. Main research topics are Spherical surface digital topology and 3D Rebuilding. Email: houmiaole@bucea.edu.cn

\section{INTRODUCTION}

The Quaternary Triangular Mesh (QTM) is an array of discrete grids. In the partition of QTM, an octahedron is used as a basis. It can simulate the surface of the Earth when it is recursively partitioned to a certain degree. QTM is efficient to deal with the global data because of its advantages of multi-resolution and hierarchy. In recent years, Scholars both at home and abroad made progress in the partition of the QTM. Dutton [1996] brought out a theory that a spherical triangle can be subdivided by the latitudes/longitudes. When a triangle is subdivided, the latitudes/longitudes pairs of any two of its three vertices are averaged to yield edge midpoint locations. Zhao Xuesheng[2001] introduced the recursive refining approach, to determine spatial relation between two spherical objects is approached by recursive refine based on QTM hierarchical partition. Zhang Shengmao[2008] analyzed the triangles which are subdivided based on QTM, the research indicated that along with the increase of the partition hierarchy, the area and change of the triangle become more stable. Sun Wenbin[2009] provided the connotation of "near-equal grid" on spherical surface, then done research on the partition method and character analysis of Near-Equal grid on spherical facet. Bai Jianjun[2011] analyzed the existent partition method of spherical surface and provided a hierarchical tessellation meshes of WGS-84 ellipsoidal surface based on QTM, and also calculated the area and side length of the multi-resolution meshes on different levels. According to the existing literature, the researches on the partition hierarchy based on QTM are mostly based on the whole sphere. However, along with the increase of the partition hierarchy, the number of triangle grids that are subdivided base on QTM is increased four times (the correspondence between the number of triangle grids and the partition hierarchy of QTM is shown in Figure 1). Though the advanced computer hardware devices contribute to improve the speed of rend of the triangle grids, the level of the computer hardware devices is limited while the data volume is limitless. As a result, the gap between the data volume and the level of the computer hardware devices is huge. Therefore, the speed of the real-time rend of the triangle grids is not satisfactory just by 
improving the level of the computer hardware devices.

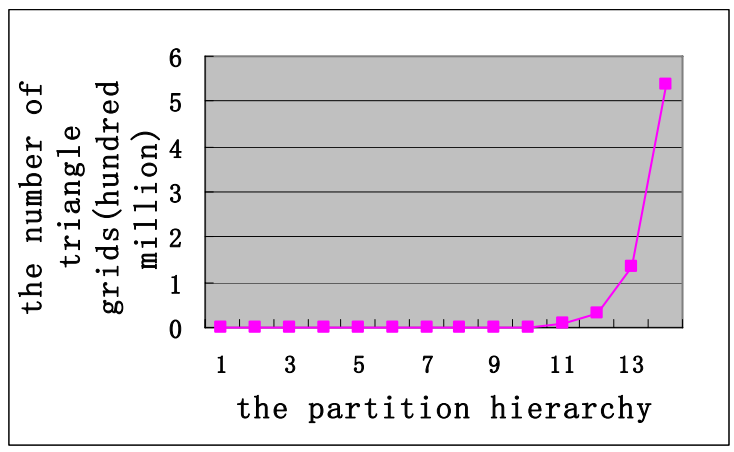

Figure 1 Correspondence between the number of triangle grids and the partition hierarchy

Spatial analysis and application based on QTM are depending on resolving the problem that improving the speed of the real-time rend of the triangle grids, that mean rending the globe fast and accurately by the way of the triangle grids. In the actual application, the range of observation is limited. The viewer may not care all area of the globe, but only interested in the part area. Therefore, building the partition model of multi-resolution LOD based on QTM, and decreasing the rending number of the triangle grids that are not in the interested area, are the effective way of improving the speed of the real-time rend of the triangle grids in the base of improving the accuracy that the triangles are subdivided base on QTM.

\section{COMPARE THE EXISTING LOD ALGORITHM}

LOD (Level of Details) technology was first introduced in 1976 by Clark, who mentioned that an object is shown by different resolutions or different levels of detail. There are three stages in the development of LOD technology, Discrete LOD; Continuous LOD; Multi-Resolution LOD.

In Discrete LOD, the models of different accuracy are built, and every model has its levels of detail. Coarse model can be used when observed from afar, while high- accuracy model can be used when observed nearly. Since different models lack of continuity, the sight will be discontinuous in the progress of rending. Continuous LOD is a method showing compact model, which can produce models of infinite different levels of detail by the way of iterative operation, so it makes the models real-time shown. The most important characteristics of Continuous LOD is that everywhere in the same model has the same level of detail, however, the area which is near from viewpoint can be seen more clearly in real life. Therefore, Continuous LOD is only applied to simplifying the single object or small scale terrain. The basic idea of Multi-Resolution LOD is that a $3 \mathrm{D}$ scene is shown by different LOD levels. The LOD level of the area depends on the distance between the area and the viewpoint, and it will be updated automatically following the viewpoint. Based on the comparison above, Multi-Resolution LOD is adopted, observers can see the coarse model when they observe afar; and they can see the highaccuracy model when they observe nearly, at the same time, it reduces the pressure of the CPU.

\section{THE SUBDIVISION OF MULTI-RESOLUTION LOD BASED ON QTM}

\subsection{Partition The QTM Into Several Blocks}

QTM is an array of triangle grids, which are subdivided by an Octahedron. When the partition level is high, the number of the triangle grids can reach tens of millions, even hundreds of millions. At present, the memory capacity and the rending performance of the computer are limited. As a result, it's worthless to compare the position between the triangle grid and the viewpoint one by one. Therefore, it's necessary to partition the model into several blocks. Theoretically, the size of the block is unlimited. Yet in practical application, it may affect the performance of the system if the size of the block is too big; otherwise, it can increase the times of data scheduling if the size of the block is too small. Thus, it's necessary to choose the appropriate number and size of the blocks. The triangle grids of QTM contain the property of latitudes/longitudes, so the model based on QTM may be divided by the way of latitudes/longitudes. According to the partition hierarchy of QTM, the number of the blocks will be dynamically chosen, and the correspondence between the partition hierarchy and the number of the blocks is shown in Figure 2. 


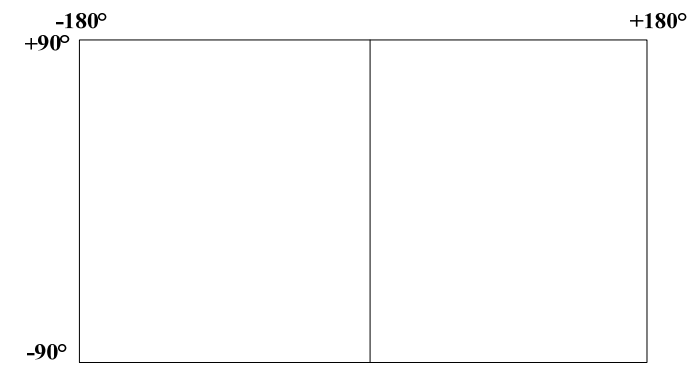

the partition hierarchy $\mathrm{N}=2$,

the number of blocks $\mathrm{T}=2$

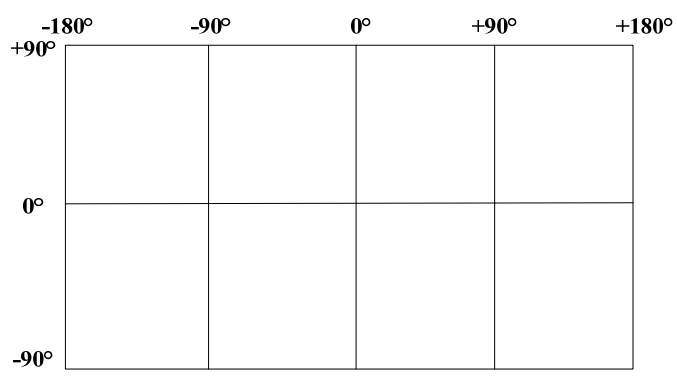

the partition hierarchy $\mathrm{N}=3$,

the number of blocks $\mathrm{T}=8$

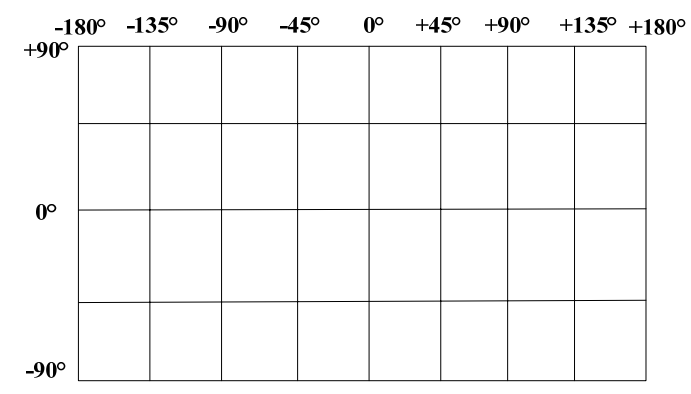

the partition hierarchy $\mathrm{N}=\mathrm{k}$,

the number of blocks $\mathrm{T}=2 * 4^{(\mathrm{k}-2)}$

Figure 2 the correspondence between the partition hierarchy and the number of the blocks

\subsection{Thinning Area}

In the progress of partitioning the QTM, it is not necessary to subdivide the whole model, but only to subdivide the block which is near form the viewpoint. The block which is the nearest form the viewpoint is the thinning area. The partition hierarchy of QTM is supposed as k, according to the block strategy, the number of the block $(\mathrm{B})$ is $2 * 4^{(\mathrm{k}-2)}$, and $\mathrm{B}=\left\{\mathrm{B}_{0}\right.$,
$\left.\mathrm{B}_{1} \ldots\right\}$, every block contain different numbers of triangle grids, that mean $\mathrm{B}_{0}=\left\{\mathrm{T}_{00}, \mathrm{~T}_{01}, \ldots\right\}, \mathrm{B}_{1}=\left\{\mathrm{T}_{10}, \mathrm{~T}_{11}, \ldots\right\}$.

The center coordinate of $\mathrm{B}_{0}$ is noted as Center $\left(\mathrm{B}_{0}\right)$, and

Center $\left(\mathrm{B}_{0}\right)$

$=\frac{\text { coordinate }\left(\mathrm{T}_{00}\right)+\operatorname{coordinate}\left(\mathrm{T}_{01}\right)+\ldots}{\operatorname{Sum}\left(\mathrm{B}_{0}\right)} \cdot$ Comparing the distance between the block and the viewpoint, the block $\mathrm{B}_{k}$ which is the nearest from the viewpoint is noted as $\operatorname{Min}\left(\mathrm{B}_{k}\right)$, and $\operatorname{Min}\left(\mathrm{B}_{k}\right)=\left\{\operatorname{Center}\left(\mathrm{B}_{0}\right)\right.$, Center $\left.\left(\mathrm{B}_{1}\right) \ldots\right\}$. The range of block $\mathrm{B}_{k}$ is the thinning area.

The triangle grids are subdivided in the thinning area on the basis of original partition hierarchy. The area which the observers don't care will keep the original partition hierarchy, while the thinning area will be subdivided again, shown in Figure 3.

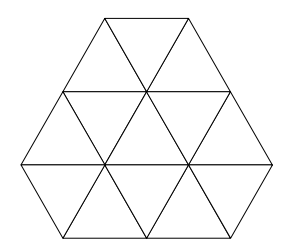

(a) the low level of the partition hierarchy

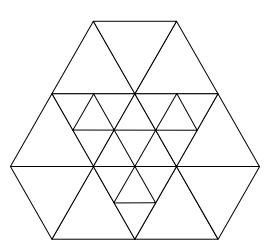

(b) the high level of the partition hierarchy in the thinning area
Figure 3 the thinning area in the QTM

Alone with the continuous movement of the viewpoint, the thinning area will be changing, the triangle grids in the thinning area will be changing as well. Whenever the viewpoint is changing, he relationship between the blocks and the viewpoint needed to judge, then the triangle grids in the thinning area will be also updated. 


\subsection{Deal With The Cracks}

The QTM grids which are different resolutions will be adjoining by the time of the partition of Multi-Resolution LOD. Computer use discrete plane to express the information of hook face, so that the sharing edge or vertex of the QTM grids which are different resolutions will not be in the same plane. If the problem is not processed, the cracks will be produced in the process of visualization. As shown in Figure 4: the resolutions of the QTM grids A and the QTM grids B,C,D are different, the cracks will be produced in the rending if we don't take steps.

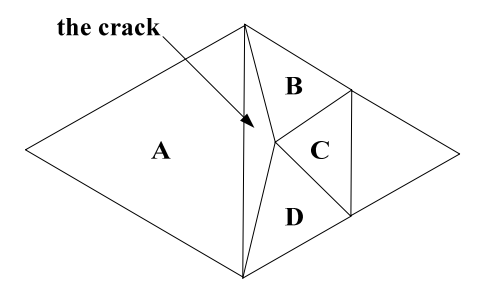

Figure 4 the cracks caused by different subdivisions

The cracks must be eliminated in order to realize seamless and visualization expressing of the model based on QTM. Scholars both at home and abroad made progress in building the seamless model based on QTM, Sun Wenbin provided the vertex extension method of eliminating the cracks in 2008 . He eliminated the cracks by the way of stretching the vertex of the higher resolution grids to the edge of the low resolution grids. The cracks caused by different subdivisions are eliminated by the way of the vertex extension in his method, it don't change the partition method of the grids. At the same time, this method realizes seamless expressing of the model based on QTM on the base of hierarchical nesting. Based on the advantages above, the vertex extension method of eliminating the cracks that caused by different subdivisions is adopted in this article.

\subsection{Algorithm Flow}

In this paper, the QTM model is firstly partitioned into sever blocks on the base of the partition hierarchy and the latitudes/longitudes of the triangle grids; the distance between the blocks and the viewpoint are compared. The block which is the nearest from the viewpoint is selected as the thinning area, and the triangle grids in the thinning area will be subdivided. Finally, the cracks caused by different subdivisions will be dealt with. The algorithm flow chart is shown in Figure 5.

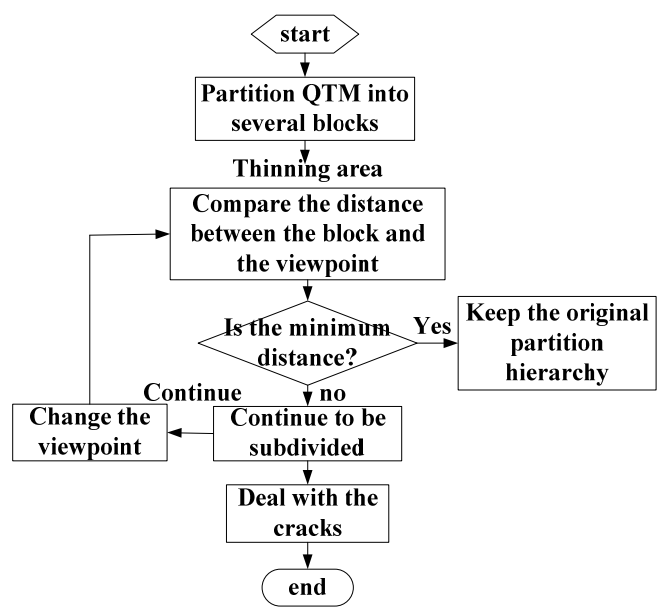

Figure 5 the algorithm flow chart

\section{EXPERIMENTS}

\subsection{Experimental Tools}

In this article, Visual C\#2008 and Direct3D are selected as development tools. Direct3D is a powerful 3D image SDK provided by Microsoft. It can provide powerful rending function in support of video card. The progress that rending the model from $3 \mathrm{D}$ space to computer screens is realized by the fixed pipeline of Direct3D.

\subsection{Experimental Effect}

In this paper, the QTM model is subdivided as seven times, and the number of the triangle grids is 32768.Then we partition the model into several blocks on the base of the latitudes/longitudes of the triangle grids. The number of blocks is 512 based on the block strategy. When the position of the viewpoint is changing, we only judge the distance between the blocks and the viewpoint, and select the block which is the nearest from the viewpoint as the thinning area. In this article, the partition hierarchy of the thinning area is increased three times on the base of the original partition hierarchy, which means that the partition hierarchy of the thinning area is nine times. If the whole globe model is subdivided, the number of the triangle grids which need rending is 524,288. However, we can realize the effect that the partition hierarchy partition hierarchy is nine, while the number of the triangle grids which need rending is only 36,800 in this paper. In a word, the number of the triangle grids that need rending is decreased on condition that 
increases the partition hierarchy of the thinning area by this method. The experiment effect is shown as follow.

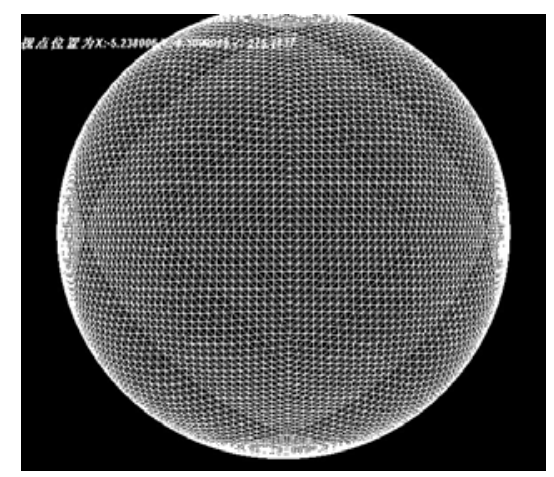

Figure 6 the partition hierarchy of QTM is six

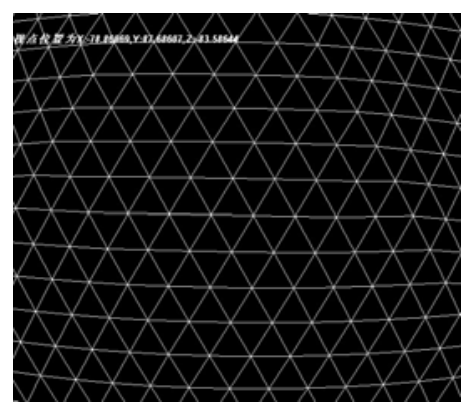

Figure 7 the effect of zoom in area

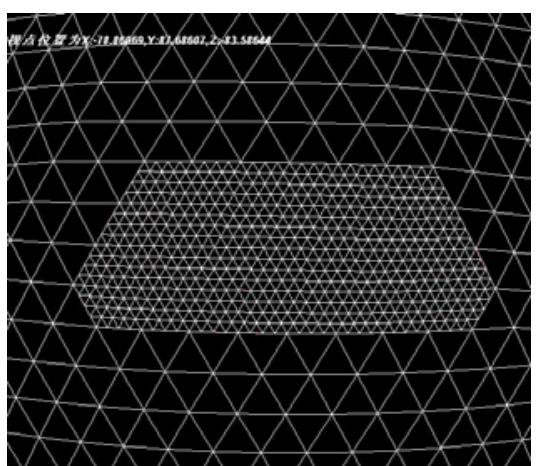

Figure 8 the partition of Multi-Resolution LOD based on QTM

\section{CONCLUSIONS}

Along with the increase of the partition hierarchy, the number of triangle grids that are subdivided base on QTM is increased four times. Huge amount of data will seriously affect the real-time and verisimilitude of the system. To resolve this problem, we provide the partition of Multi-Resolution LOD based on QTM; this method not only has the advantages of blocks, but also avoids a large number of pretreatment works, and it increases the partition hierarchy of the thinning area on the base of rending smoothly. As a result, this paper sets the stage for building the high revolution image model and terrain model.

\section{REFERENCES}

[1] Bartholdi III, Goldsman P. Continuous indexing of hierarchical subdivisions of the globe. International Journal of Geographical Information Science, 2001, 15(6): 489-522.

[2] DUTTON G. Encoding and Handling Geospatial Data with Hierarchical Triangular Meshes [A ].Proceeding of 7th International Symposium on Spatial Data Handling[C ]. Netherlands: Delft University of Techno logy, 1996. 34-43.

[3] Zhao, X. SH., and Chen, J. Hierarchical Spatial Relation Reasoning Based on Spherical O-QTM Partition. Acta Geodaetica et Cartographica Sinica,2001,30(4):355-360(in Chinese).

[4] Zhang, SH. M. Research of Global Remote Sensing Image Browser System Based on Octahedron Discrete Global Grids Model. Ph.D.Dissertation of EAST CHINA NORMAL UNIVERCITY, 2009,132pp(in Chinese)

[5] Sun, W. B., Zhao, X. SH., Gao, Y. L., Wang, H. B., Partition Methods and Character Analysis of Near-Equal Grids on Spherical Facet, Geography and Geo-Information Science, 2009,25(1):53-56(in Chinese).

[6] Bai, J. J., Sun, W. B., Zhao, X. SH., Character Analysis and Hierarchical Partition of WGS-84 Ellipsoidal Facet Based on QTM, Acta Geodaetica et Cartographica Sinica,2011,40(2):243-248(in Chinese).

[7] Clark James. Hierarchical Geometric Models for Visible Surface Algorithms [J].Communications of the ACM. 19(10),PP.547—554.1976. 
International Archives of the Photogrammetry, Remote Sensing and Spatial Information Sciences, Volume XXXVIII-4/W25, 2011 ISPRS Guilin 2011 Workshop, 20-21 October 2011, Guilin, China

[8] Du, Y., A Research on Key Technologies for Global Multi-resolution Virtual Terrain Environment. Ph.D.Dissertation of The PLA Information Engineering University, 2005,173pp(in Chinese).
[9] Sun, W. B., Zhao, X. SH., A Hierarchical Seamless Model of Spatial Data Based on QTM, Journal of China University of Mining \& Technology 2008,37(5):675-679(in Chinese). 